\title{
Erratum to: Cockroach, tick, storage mite and other arthropod allergies: Where do we stand with molecular allergy diagnostics? Part 15 of the Series Molecular Allergology.
}

Erratum to: Hilger C, Kuehn A, Raulf M, Jakob T. Cockroach, tick, storage mite and other arthropod allergies: Where do we stand with molecular allergy diagnostics? Part 15 of the Series Molecular Allergology. Allergo J Int 2014;23:172-8

DOI: $10.1007 / s 40629-014-0024-2$

In table 3 "Individual allergens of other arthropods identified according to the IUIS Allergen Nomenclature Sub-Committee" (p. 176) in the segment Chi-

German version www.springermedizin.de/ allergo-journal ronomus thummi thummi (red chironomid larvae)" the haemoglobin component I/IA is attributed to the allergen Chi t 2, not to the allergen Chi t 1 . The correct segment of table 3 should read as follows.
Individual allergens of other arthropods identified according to the IUIS Allergen Nomenclature Sub-Committee

Allergen
Chiro
thum
(Red

Name

Molecular weight $(\mathrm{kDa})$
Table 3

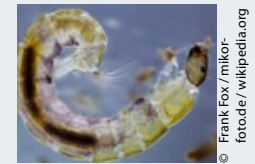

Haemoglobin component III/IV 16 Haemoglobin component I/IA $\quad 16$ Haemoglobin components II- $\beta, \mathrm{VI}, 16$ VIII, IX

Haemoglobin component IIIA $\quad 16$ Haemoglobin component $\mathrm{X}$
Christiane Hilger, PhD

Annette Kuehn

Laboratoire d'Immunogénétique et d'Allergologie

Centre de Recherche Public de la Santé, Luxembourg, Luxembourg

E-Mail: christiane.hilger@crp-sante.lu

\section{Monika Raulf}

Institute for Prevention and Occupational Medicine of the German Statutory Accident Insurance, Institute of the Ruhr University Bochum (IPA)

\section{Thilo Jakob}

Allergy Research Group, Department of Dermatology, Medical Center - University of Freiburg, Germany

\section{Cite this as}

Hilger C, Kuehn A, Raulf M, Jakob T. Erratum to: Cockroach, tick, storage mite and other arthropod allergies: Where do we stand with molecular allergy diagnostics? Part 15 of the Series Molecular Allergology. Allergo J Int 2014;23:281

DOI: $10.1007 / \mathrm{s} 40629-014-0035-z$ 